\title{
Robust Control of Underactuated Systems: Higher Order Integral Sliding Mode Approach
}

\author{
Sami ud Din, ${ }^{1,2}$ Qudrat Khan, ${ }^{3,4}$ Fazal ur Rehman, ${ }^{1}$ and Rini Akmeliawati ${ }^{3}$ \\ ${ }^{1}$ Department of Electrical Engineering, Capital University of Science and Technology (CUST), \\ Kahuta Road, Express Highway, Islamabad 44000, Pakistan \\ ${ }^{2}$ Department of Electrical Engineering, The University of Lahore (UOL), Japan Road, Express Highway, Islamabad 44000, Pakistan \\ ${ }^{3}$ Department of Mechatronics Engineering, International Islamic University, 50728 Kuala Lumpur, Malaysia \\ ${ }^{4}$ Center for Advanced Studies in Telecommunications, COMSATS Institute of Information Technology, Islamabad 44000, Pakistan
}

Correspondence should be addressed to Sami ud Din; engrsamiuddin@gmail.com

Received 25 September 2015; Revised 8 January 2016; Accepted 12 January 2016

Academic Editor: Wenguang Yu

Copyright (C) 2016 Sami ud Din et al. This is an open access article distributed under the Creative Commons Attribution License, which permits unrestricted use, distribution, and reproduction in any medium, provided the original work is properly cited.

\begin{abstract}
This paper presents a robust control design for the class of underactuated uncertain nonlinear systems. Either the nonlinear model of the underactuated systems is transformed into an input output form and then an integral manifold is devised for the control design purpose or an integral manifold is defined directly for the concerned class. Having defined the integral manifolds discontinuous control laws are designed which are capable of maintaining sliding mode from the very beginning. The closed loop stability of these systems is presented in an impressive way. The effectiveness and demand of the designed control laws are verified via the simulation and experimental results of ball and beam system.
\end{abstract}

\section{Introduction}

The control design of underactuated systems was the main focus of the researchers in the current and last decade. These systems, by definition, contain less number of control inputs/actuators as compared to the degree of freedom [1]. This feature makes them quite different from the other nonlinear plants where the systems operate with the same number of inputs and outputs, the so-called fully actuated systems. The control design of these systems is quite demanding because of their vital theoretical and practical applications in the areas of aerospace systems, marine systems, humanoids, locomotive systems, manipulators of different kinds, and so forth [2]. This family also includes ball and beam system [3], TORA (translational oscillator with rotational actuator) [4], and inverted pendulum system [5]. These systems are used in order to have minimum weight, cost, and energy usage while still retaining the key features of the processes. In addition, another significant feature of underactuated systems is less damage in case of collision with other objects which in turn provides more safety to actuators [6]. Underactuation can be raised due to the hardware failure; this hardware solution to actuator failures can be achieved by equipping the vehicle with redundant actuators [2]. Note that, in case of fully actuated systems, there exists a broad range of design techniques in order to improve performance and robustness. These include adaptive control, optimal control, feedback linearization, and passivity. However, it may be difficult to apply such techniques in large class of underactuated systems because sometimes these systems are not linearizable using smooth feedback [7] also due to the existence of unstable hidden modes in some systems. Brockett [8] also provided a necessary condition for the hold of stable smooth feedback law, but this condition is not satisfied in the majority of underactuated systems. Nevertheless, control design experts have employed approximate feedback linearization [9-11] and backstepping control [12]. Passivity-based methodology is also used to control such systems but the main drawback in this technique is its narrow range of applications [13]. Sliding mode control is also proposed for the class of underactuated systems [6] but the problem with sliding mode control is presence of chattering. 
The aforementioned design strategies were quite suitable and resulted in satisfactory results but it is worthy to note that the system often becomes too sensitive to disturbance in the reaching phase of sliding mode strategy that the system may even become unstable. Therefore, in order to get rid of this issue the integral sliding mode strategy was proposed [14-16]. In this paper a robust integral sliding mode control (RISMC) approach for underactuated systems is proposed. The benefit of this strategy is enhancement of robustness from initial time instant. It also suppresses the well-known chattering phenomenon across the manifold. Before the design presentation, the system is suitably transformed into special formats. An integral sliding mode strategy is proposed for both the cases along with their comprehensive stability analysis. The proposed technique is practically implemented on the ball and beam system to authenticate the affectivity and efficiency of the designed algorithm. Note that in this paper our contributions are twofold. The first one is the development of the RISMC and the second one is the practical results of the system on the said system. The rest of the paper is organized as follows. In Section 2, the problem is formulated into two special formats which further simplify the design methodology. In Section 3, the integral sliding mode strategy for both the cases is discussed in detail accompanied by their respective stability analysis in terms of Lyapunov theory. Section 4 presents the development of the control laws, simulation, and practical results of the ball and beam system. Section 5 concludes the overall efforts being made in this study. In the end more relevant recent articles are enlisted.

\section{Problem Formulation}

The dynamic equations which govern the motion of the class of underactuated system can be presented as

$$
\begin{aligned}
& J(q) \ddot{q}+C(q, \dot{q}) \dot{q}+G(q)+F(\dot{q}) \\
& =B(\tau+\delta(q, \dot{q}, t)),
\end{aligned}
$$

where $q, \dot{q}$, and $\ddot{q}$ are $n$-dimensional position, velocity, and acceleration vectors and $J(q), C(q, \dot{q}), G(q)$, and $F(\dot{q})$ represent the inertia, Coriolis, gravitational, and fractional torques matrices, respectively. $\tau$ is the measured control input, and $\delta(q, \dot{q}, t)$ represents the uncertainties in the control input channel whereas $B$ is the control input channel.

It is assumed that $\operatorname{rank}\left(J^{-1}(q) B\right)=m$ and the origin is considered to be the equilibrium point for the aforementioned system. Now, the system in (1) can be rewritten in alternate form as follows:

$$
\begin{aligned}
& m_{11}(q) \ddot{q}_{1}+m_{12}(q) \ddot{q}_{2}+h_{1}(q, \dot{q})=0, \\
& m_{21}(q) \ddot{q}_{1}+m_{12}(q) \ddot{q}_{2}+h_{2}(q, \dot{q})=\tau,
\end{aligned}
$$

where $q=\left[q_{1}, q_{2}\right]^{T}$ represents the states of the system and $q$ and $\dot{q}$ point to the states. In order to design a control law, the system in (2) can be transformed into two formats which are described in the subsequent study.
2.1. System in Cascaded Form. Following some algebraic manipulations, the system in (2) may be written in cascaded form as follows [17]:

$$
\begin{aligned}
& \dot{x_{1}}=x_{2}+d_{1}, \\
& \dot{x}_{2}=f_{1}\left(x_{1}, x_{2}, x_{3}, x_{4}\right)+d_{2}, \\
& \dot{x_{3}}=x_{4}, \\
& \dot{x_{4}}=f_{2}\left(x_{1}, x_{2}, x_{3}, x_{4}\right)+b\left(x_{1}, x_{2}, x_{3}, x_{4}\right) \tau+d_{3},
\end{aligned}
$$

where $x_{1}, x_{2}, x_{3}, x_{4}$ are measurable states of the systems such that $x_{1}$ and $x_{2}$ are pointing to the position and velocity of the indirect actuated system (3) while $x_{3}$ and $x_{4}$ represent the position and velocity of the directly actuated system (4). $\tau$ represents the controlled signal, as already discussed, to the system (4) input. Owing to the assumption stated immediately after (1), the inverse of $b$ exists. The nonlinear functions $f_{1}, f_{2}: R^{4 n} \rightarrow R^{n}, b: R^{4 n} \rightarrow R^{n \times n}$ are smooth in nature. Now, following the procedure of [6], the disturbances $d_{1} d_{2} d_{3}$ are deliberately introduced to get an approximate controllable canonical form. Note that practical systems like inverted pendulum [18], TORA [4], VTOL (vertical take-off and landing) aircraft [17], and quad rotor [19] can be put in the form presented in (3) and (4). Before proceeding to the control design of the above cascaded form, the following assumptions are made.

Assumption 1. Assume that

$$
f_{1}(0,0,0,0)=0 .
$$

This condition is necessary for the system origin to be in equilibrium point when the system is operated in closed loop.

Assumption 2. $\partial f_{1} / \partial x_{3}$ is invertible or $\partial f_{1} / \partial x_{4}$ is invertible.

Assumption 3. $f_{1}\left(0,0, x_{3}, x_{4}\right)=0$ is an asymptotically stable manifold, that is, $x_{3}$, and $x_{4}$ approaches zero.

Note that Assumptions 2 and 3 lie in the category of nonnecessary conditions. These are only used when one needs to furnish the closed loop system with a sliding mode controller (see for details [6]).

2.2. Input Output Form. The system in (3) and (4) can be transformed into the following input output form while following the procedure reported in [16]. Let us assume that the system has a nonlinear output $y=h(x)$. To this end we denote

$$
\begin{aligned}
& L_{f} h(x)=\frac{\partial h(x)}{\partial x} f(x)=\nabla h(x) f(x), \\
& L_{f_{\tau}} h(x)=\frac{\partial h(x)}{\partial x} f_{\tau}=\nabla h(x) f_{\tau} .
\end{aligned}
$$

Recursively, it can be written as

$$
\begin{aligned}
& L_{f}^{0} h(x)=h(x), \\
& L_{f}^{j} h(x)=L_{f}\left(L_{f}^{j-1} h(x)\right)=\nabla\left(L_{f}^{j-1} h(x)\right) f(x) .
\end{aligned}
$$


Assume that the system reported in (3)-(4) has a relative degree " $r$ " with respect to the defined nonlinear output. Therefore, owing to [20], one has

$$
y^{(r)}=L_{f}^{r} h(x)+L_{g}\left(L_{f}^{r-1} h(x)\right) \tau+\zeta(x, t)
$$

subject to the following conditions:

(1) $L_{g}\left(L_{f}^{i} h(x)\right)=0 \forall x \in B$, where $B$ indicates the neighborhood of $x_{0}$ for $i<r-1$;

(2) $L_{g}\left(L_{f}^{r-1} h(x)\right) \neq 0$, where $\zeta(x, t)$ represents the matched unmodeled uncertainties. System (8), by defining the transformation $y^{(i-1)}=\xi_{i}$ [21], can be put in the following form:

$$
\begin{gathered}
\dot{\xi}_{1}=\xi_{2}, \\
\dot{\xi}_{2}=\xi_{3}, \\
\vdots \\
\dot{\xi}_{n}=\varphi(\widehat{\xi}, \widehat{\tau})+\gamma(\widehat{\xi})\left\{\tau+\Delta G_{m}(\widehat{\xi}, \widehat{\tau}, t)\right\},
\end{gathered}
$$

where the transformed states $\widehat{\xi}=\left(\xi_{1}, \xi_{2}, \ldots, \xi_{n}\right)$ are phase variables, $\tau$ is the control input, and $\Delta G_{m}(\widehat{\xi}, \widehat{\tau}, t)$ represents matched uncertainties. It is worthy to notice that the inverted pendulum and the ball and beam systems can be replaced in the aforementioned form.

Note that both the formats are ready to design the control law for these systems. In the next section, we outline the design procedure for both the forms.

\section{Control Law Design}

The control design for the forms presented in (3)-(4) and (9) is carried out in this section which we claim as our main contribution in this paper. The main objective in this work is to enhance the robustness of the system from the very beginning of the process which is the beauty of integral sliding mode control. In general, the integral sliding mode control law appears as follows [14]. In the subsequent subsections, the authors aim to present the design procedure.

3.1. Integral Sliding Mode. This variant of sliding mode possesses the main features of the sliding mode like robustness and the existence chattering across the switching manifold. On the other hand, the sliding mode occurs from the very start which, consequently, provides insensitivity of disturbance from the beginning. The control law can be expressed as follows:

$$
\tau=\tau_{0}+\tau_{1},
$$

where the first component on the right hand side of (10) governs the systems dynamics in sliding modes whereas the second component compensates the matched disturbances. Now, the aim is to present the design of the aforesaid control components.
3.1.1. Control Design for Case-1. This control design for case1 is the main obstacle in this subsection. To define both the components, the following terms are defined:

$$
\begin{aligned}
& e_{1}=x_{1}, \\
& e_{2}=x_{2}, \\
& e_{3}=f_{1}\left(x_{1}, x_{2}, x_{3}, x_{4}\right), \\
& e_{4}=\frac{\partial f_{1}}{\partial x_{1}} x_{2}+\frac{\partial f_{1}}{\partial x_{2}} f_{1}+\frac{\partial f_{1}}{\partial x_{3}} x_{4} .
\end{aligned}
$$

Using these new variables, the components of the controller are designed in the following subsection. For the sake of completeness the design of this component is worked out via simple pole placement. Following the design procedure of pole placement method, one gets

$$
\tau_{0}=-k_{1} e_{1}-k_{2} e_{2}-k_{3} e_{3}-k_{4} e_{4},
$$

where $k_{i} i=1,2,3,4$ are the gains of this control component. This control component steers the states of the nominal system to their defined equilibrium. Now, in the subsequent study the design of the uncertainties compensating term is presented. An integral manifold is defined as follows:

$$
\sigma=c_{1} e_{1}+c_{2} e_{2}+c_{3} e_{3}+e_{4}+z=\sigma_{0}+z \text {, }
$$

where $\sigma_{0}=c_{1} e_{1}+c_{2} e_{2}+c_{3} e_{3}+e_{4}$ represents the conventional sliding manifold which is Hurwitz by definition.

Now, computing $\dot{\sigma}$ along (3)-(4), one has

$$
\begin{aligned}
\dot{\sigma}= & c_{1}\left(x_{2}+d_{1}\right)+c_{2}\left(f_{1}\left(x_{1}, x_{2}, x_{3}, x_{4}\right)+d_{2}\right) \\
& +c_{3}\left(\frac{d f_{1}\left(x_{1}, x_{2}, x_{3}, x_{4}\right)}{d t}\right)+\frac{d}{d t}\left(\frac{\partial f_{1}}{\partial x_{1}} x_{2}\right) \\
& +\frac{\partial f_{1}}{\partial x_{1}} \dot{x_{2}}+\frac{d}{d t}\left(\frac{\partial f_{1}}{\partial x_{2}} f_{1}\right)+\frac{\partial f_{1}}{\partial x_{2}} \dot{f}_{1} \\
& +\frac{d}{d t}\left(\frac{\partial f_{1}}{\partial x_{3}} x_{4}\right)+\frac{\partial f_{1}}{\partial x_{3}} f_{2}\left(x_{1}, x_{2}, x_{3}, x_{4}\right) \\
& +\frac{\partial f_{1}}{\partial x_{3}} b\left(x_{1}, x_{2}, x_{3}, x_{4}\right) \tau_{0} \\
& +\frac{\partial f_{1}}{\partial x_{3}} b\left(x_{1}, x_{2}, x_{3}, x_{4}\right) \tau_{1}+\frac{\partial f_{1}}{\partial x_{3}} d_{3} .
\end{aligned}
$$

Now, choose the dynamics of the integral term as follows:

$$
\begin{aligned}
\dot{z}= & -c_{1} x_{2}-c_{2} f_{1}\left(x_{1}, x_{2}, x_{3}, x_{4}\right) \\
& -c_{3}\left(\frac{d f_{1}\left(x_{1}, x_{2}, x_{3}, x_{4}\right)}{d t}\right)-\frac{d}{d t}\left(\frac{\partial f_{1}}{\partial x_{1}} x_{2}\right) \\
& -\frac{\partial f_{1}}{\partial x_{1}} \dot{x_{2}}-\frac{d}{d t}\left(\frac{\partial f_{1}}{\partial x_{2}} f_{1}\right)-\frac{\partial f_{1}}{\partial x_{2}} \dot{f}_{1} \\
& -\frac{d}{d t}\left(\frac{\partial f_{1}}{\partial x_{3}} x_{4}\right)-\frac{\partial f_{1}}{\partial x_{3}}\left(x_{1}, x_{2}, x_{3}, x_{4}\right) \tau_{0} .
\end{aligned}
$$


The expression of the term which compensates the uncertainties may be written as follows:

$$
\begin{aligned}
\tau_{1}= & -\left(\frac{\partial f_{1}}{\partial x_{3}} b\left(x_{1}, x_{2}, x_{3}, x_{4}\right)\right)^{-1} \\
& \cdot\left(\frac{\partial f_{1}}{\partial x_{3}} f_{2}\left(x_{1}, x_{2}, x_{3}, x_{4}\right)+K \operatorname{sign}(\sigma)\right) .
\end{aligned}
$$

The overall controller will look like

$$
\begin{aligned}
\tau=- & k_{1} e_{1}-k_{2} e_{2}-k_{3} e_{3}-k_{4} e_{4} \\
- & \left(\frac{\partial f_{1}}{\partial x_{3}} b\left(x_{1}, x_{2}, x_{3}, x_{4}\right)\right)^{-1} \\
& \cdot\left(\frac{\partial f_{1}}{\partial x_{3}} f_{2}\left(x_{1}, x_{2}, x_{3}, x_{4}\right)+K \operatorname{sign}(\sigma)\right) .
\end{aligned}
$$

The constants $c_{i}$ 's are control gains which are selected intelligently according to bounds. In the forthcoming paragraph, the stability of the presented integral sliding mode is carried out in the presence of the disturbances and uncertainties. Consider the following Lyapunov candidate function:

$$
V=\frac{1}{2} \sigma^{2}
$$

The time derivative of this function along dynamics (11) becomes

$$
\begin{aligned}
\dot{V} & =\sigma \dot{\sigma}=\sigma\left(c_{1}\left(x_{2}+d_{1}\right)\right. \\
& +c_{2}\left(f_{1}\left(x_{1}, x_{2}, x_{3}, x_{4}\right)+d_{2}\right) \\
& +c_{3}\left(\frac{d f_{1}\left(x_{1}, x_{2}, x_{3}, x_{4}\right)}{d t}\right)+\frac{d}{d t}\left(\frac{\partial f_{1}}{\partial x_{1}} x_{2}\right) \\
& +\frac{\partial f_{1}}{\partial x_{1}} \dot{x_{2}}+\frac{d}{d t}\left(\frac{\partial f_{1}}{\partial x_{2}} f_{1}\right)+\frac{\partial f_{1}}{\partial x_{2}} \dot{f}_{1}+\frac{d}{d t}\left(\frac{\partial f_{1}}{\partial x_{3}} x_{4}\right) \\
& +\frac{\partial f_{1}}{\partial x_{3}} f_{2}\left(x_{1}, x_{2}, x_{3}, x_{4}\right)+\frac{\partial f_{1}}{\partial x_{3}}\left(x_{1}, x_{2}, x_{3}, x_{4}\right) \tau_{0} \\
& \left.+\frac{\partial f_{1}}{\partial x_{3}} b\left(x_{1}, x_{2}, x_{3}, x_{4}\right) \tau_{1}+\frac{\partial f_{1}}{\partial x_{3}} d_{3}\right) .
\end{aligned}
$$

The substitution of (15)-(16) results in the following form:

$$
\begin{aligned}
\dot{V} & \leq-|\sigma| \eta_{1}<0 \\
\text { or } \dot{V}+\sqrt{2} \eta_{1} \sqrt{V} & <0
\end{aligned}
$$

subject to $K \geq\left[\left\|\left(\partial f_{1} / \partial x_{3}\right) d_{3}+c_{1} d_{1}+c_{2} d_{2}\right\|+\eta\right]$.

This expression confirms the enforcement of the sliding mode from the very beginning of the process, that is, $\sigma \rightarrow 0$ in finite time. Now, we proceed to the actual system's stability. If one considers $e_{1}$ as the output of the system, then $e_{2}, e_{3}$, and $e_{4}$ become the successive derivatives of $e_{1}$. Whenever $\sigma=0$ is achieved, the dynamics of the transformed system
(11) will converge asymptotically to zero under the action of the control component (12) [22]. That is, in closed loop, the transformed system dynamics will be operated under (12) as follows:

$$
\left[\begin{array}{l}
\dot{e_{1}} \\
\dot{e_{2}} \\
\dot{e_{3}} \\
\dot{e_{4}}
\end{array}\right]=\left[\begin{array}{rrrr}
0 & 1 & 0 & 0 \\
0 & 0 & 1 & 0 \\
0 & 0 & 0 & 1 \\
-k_{1} & -k_{2} & -k_{3} & -k_{4}
\end{array}\right]\left[\begin{array}{l}
e_{1} \\
e_{2} \\
e_{3} \\
e_{4}
\end{array}\right]
$$

and the disturbances will be compensated via (16).

The asymptotic convergence of $e_{1}, e_{2}, e_{3}$, and $e_{4}$ to zero means the convergence of the indirectly actuated system (3) to zero. On the other hand, the states of the directly actuated system (4) will remain bounded; that is, state of (4) will have some nonzero value in order to keep $e_{1}$ at zero. Thus, the overall system is stabilized and the desired control objective is achieved.

3.2. Control Design for Case-2. The nominal system related to (9) can be replaced in the subsequent alternative form

$$
\begin{gathered}
\dot{\xi}_{1}=\xi_{2} \\
\dot{\xi}_{2}=\xi_{3} \\
\vdots \\
\dot{\xi}_{r}=\chi(\widehat{\xi}, \tau)+\tau,
\end{gathered}
$$

where $\chi(\widehat{\xi}, \tau)=\varphi(\xi, \tau)+(\gamma(\widehat{\xi})-1) \tau$. It is assumed that $\chi\left(\widehat{\xi}, \widehat{\tau}, \tau^{(k)}\right)=0$ at $t=0$ in addition to the next supposition that $(22)$ is governed by $\tau_{0}$ :

$$
\begin{gathered}
\dot{\xi}_{1}=\xi_{2} \\
\dot{\xi}_{2}=\xi_{3} \\
\vdots \\
\dot{\xi}_{r}=\tau_{0}
\end{gathered}
$$

or

$$
\dot{\xi}=A \xi+B \tau_{0}
$$

where

$$
\begin{aligned}
& A=\left[\begin{array}{cc}
0_{(r-1) \times 1} & I_{(r-1) \times(r-1)} \\
0_{1 \times 1} & 0_{1 \times(r-1)}
\end{array}\right], \\
& B=\left[\begin{array}{c}
0_{(r-1) \times 1} \\
1
\end{array}\right] .
\end{aligned}
$$


Once again, following the pole placement procedure, one may have, for the sake of simplicity, the input $\tau_{0}$ which is designed via pole placement, that is,

$$
\tau_{0}=-K_{0}^{T} \xi
$$

Now to get the desired robust performance, the following sliding manifold of integral type [14] is defined:

$$
\sigma(\xi)=\sigma_{0}(\xi)+z
$$

where $\sigma_{0}(\xi)$ is the usual sliding surface and $z$ is the integral term. The time derivative of (27) along (9) yields

$$
\begin{aligned}
& \dot{z}=-\left(\sum_{i=1}^{r-1} c_{i} \xi_{i+1}+\tau_{0}\right), \\
& z(0)=-\sigma_{0}(\xi(0)), \\
& \tau_{1}=\frac{1}{\gamma(\widehat{\xi})}\left(-\varphi(\widehat{\xi}, \tau)-(\gamma(\widehat{\xi})-1) \tau_{0}-K \operatorname{sign} \sigma\right) .
\end{aligned}
$$

This control law enforces sliding mode along the sliding manifold defined in (27). The constant $K$ can be selected according to the subsequent stability analysis.

Thus, the final control law becomes

$$
\begin{aligned}
\tau_{1} & \\
= & -K_{0}^{T} \xi \\
& +\frac{1}{\gamma(\widehat{\xi})}\left(-\varphi(\widehat{\xi}, u)-(\gamma(\widehat{\xi})-1) \tau_{0}-K \operatorname{sign} \sigma\right) .
\end{aligned}
$$

Theorem 4. Consider that $\left|\Delta G_{m}(\widehat{y}, \widehat{u}, t)\right| \leq \beta_{1}$ are satisfied; then the sliding mode against the switching manifold $\sigma=0$ can be ensured and one has

$$
K \geq\left[K_{M} \beta_{1}+\eta_{1}\right]
$$

where $\eta_{1}$ is a positive constant.

Proof. To prove that the sliding mode can be enforced in finite time, differentiating (22) along the dynamics of (3)-(4), and then substituting (30), one has

$$
\begin{aligned}
\dot{\sigma}(\xi)= & \sum_{i=1}^{r-1} c_{i} \xi_{i+1}+\tau_{0}-K \operatorname{sign} \sigma+\gamma(\widehat{\xi}) \Delta G_{m}(\widehat{\xi}, \widehat{\tau}, t) \\
& +\dot{z}
\end{aligned}
$$

Substituting (28) in (32), and then rearranging, one obtains

$$
\dot{\sigma}(\xi)=-K \operatorname{sign} \sigma+\gamma(\widehat{\xi}) \Delta G_{m}(\widehat{\xi}, \widehat{\tau}, t) .
$$

Now, the time derivative of the Lyapunov candidate function $V=(1 / 2) \sigma^{2}$, with the use of the bounds of the uncertainties, becomes

$$
\dot{V} \leq-|\sigma|\left[-K+\left|\gamma(\widehat{\xi}) \Delta G_{m}(\widehat{\xi}, \widehat{\tau}, t)\right|\right] .
$$

This expression may also be written as

$$
\begin{aligned}
\dot{V} & \leq-|\sigma| \eta_{1}<0 \\
\text { or } \dot{V}+\sqrt{2} \eta_{1} \sqrt{V} & <0,
\end{aligned}
$$

provided that

$$
K \geq\left[K_{M} \beta_{1}+\eta_{1}\right] .
$$

The inequality in (35) presents that $\sigma(\xi)$ approaches zero in a finite time $t_{s}[23]$, such that

$$
t_{s} \leq \sqrt{2} \eta_{1}^{-1} \sqrt{V}(\sigma(0))
$$

which completes the proof.

\section{Illustrative Example}

The control algorithms presented in Section 3 are applied to the control design of a ball and beam system. The assessment of the proposed controller, for the ball and beam system, is carried out on the basis of output tracking, robustness enhancement via the elimination of reaching phase, and chattering-free control input in the presence of uncertainties.

4.1. Description of the Ball and Beam System. The ball and beam system is a very sound candidate of the class of underactuated nonlinear system. It is famous because of its nonlinear nature and due to its wide range of applications in the existing era like passenger cabin balancing in luxury cars, balancing of liquid fuel in vertical take-off objects. In terms of control scenarios, it is an ill-defined relative degree system which, to some extent, does not support input output linearization. A schematic diagram with their typical parameters of the ball and beam system is displayed in the adjacent Figure 1 and Table 1, respectively. In this study the authors use the equipment manufacture by GoogolTech. In general this system is equipped with a metallic ball, which is let free to roll on a rod having a specified length, having one end fixed and the other end moved up and down via an electric servomotor. The position of the ball can be measured via different techniques. The measured position is used as feedback to the system and accordingly the motor moves the beam to balance the ball at user defined location.

The motion governing equations of this system are given below which are adopted from [24]:

$$
\begin{aligned}
& \left(m r^{2}+C_{1}\right) \ddot{\beta}+\left(2 m r \dot{r}+C_{2}\right) \dot{\beta} \\
& +\left(m g r+\frac{L}{2} M g\right) \cos \beta=\tau, \\
& C_{4} \ddot{r}-r \dot{\beta}^{2}+g \sin \beta=0,
\end{aligned}
$$




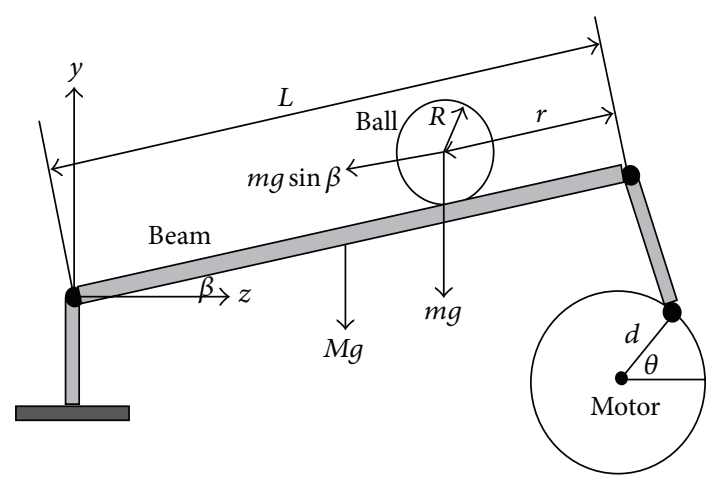

Figure 1: Schematic diagram of the ball and beam system.

where $\theta(t)$ angle is subtended to make the ball stable, the lever angle is represented by $\beta(t), r(t)$ is the position of the ball on the beam, and $v_{\text {in }}(t)$ is the input voltage of the motor whereas the controlled input appears mathematically via the expression $\tau(t)=C_{3} v_{\text {in }}(t)$ in the dynamic model.

The derived parameters used in the dynamic model of this system are represented by $C_{1}, C_{2}, C_{3}$, and $C_{4}$ with the following mathematical relations [25]:

$$
\begin{aligned}
& C_{1}=\frac{R_{m} \times J_{m} \times L}{C_{m} \times C_{b} \times d}+J_{1}, \\
& C_{2}=\frac{L}{d}\left(\frac{C_{m} \times C_{b}}{R_{m}}+C_{b}+\frac{R_{m} \times J_{m}}{C_{m} \times C_{g}}\right),
\end{aligned}
$$

$$
\begin{aligned}
& C_{3}=1+\frac{C_{m}}{R_{m}}, \\
& C_{4}=\frac{7}{5} .
\end{aligned}
$$

The equivalent state space model of this is described as follows by assuming $x_{1}=r$ (position of ball), $x_{2}=\dot{r}$ (rate of change of position), $x_{3}=\beta$ (beam angle), and $x_{4}=\dot{\beta}$ (the rate of change of angle of the motor):

$$
\begin{aligned}
\dot{x}_{1} & =x_{2}, \\
\dot{x_{2}} & =\frac{1}{C_{4}}\left(-g \sin \left(x_{3}\right)\right), \\
\dot{x}_{3} & =x_{4}, \\
\dot{x_{4}} & =\frac{1}{m x_{1}^{2}+C_{1}}\left(\tau-\left(2 m x_{1} x_{2}+C_{2}\right) x_{4}\right. \\
& \left.-\left(m g x_{1}+\frac{L}{2} M g\right) \cos x_{3}\right) .
\end{aligned}
$$

Now, the output of interest is $y=x_{1}$, which represents the position of the ball. This representation is similar to that reported in (3)-(4). In the next discussion the controller design is outlined.

4.2. Controller Design. Following the procedure outlined in Section 3, the authors proceed as follows:

$$
\begin{aligned}
y & =x_{1}, \\
\dot{y} & =x_{2}, \\
\ddot{y} & =-\frac{g}{C_{4}} \sin \left(x_{3}\right), \\
y^{(3)} & =-\frac{g}{C_{4}} x_{4} \cos \left(x_{3}\right), \\
y^{(4)} & =\frac{1}{C_{4}\left(m x_{1}^{2}+C_{1}\right)}\left[-\tau \cos x_{3}+\left(2 m x_{1} x_{2}+C_{2}\right) x_{4} \cos x_{3}+\left(m g x_{1}+\frac{L}{2} M g\right) \cos ^{2} x_{3}+x_{4}^{2}\left(m x_{1}^{2}+C_{1}\right) \sin x_{3}\right], \\
y^{(4)} & =f_{s}+h_{s} \tau, \\
f_{s} & =\frac{g}{C_{4}}\left[\frac{\left(2 m x_{1} x_{2}+C_{2}\right) x_{4}+\left(m g x_{1}+(L / 2) M g\right) \cos ^{2} x_{3}+x_{4}^{2} \sin x_{3}}{m x_{1}^{2}+C_{1}}\right], \\
h_{s} & =\frac{-g \cos x_{3}}{C_{4}\left(m x_{1}^{2}+C_{1}\right)} .
\end{aligned}
$$


TABLE 1: Parameters and values used in equations.

\begin{tabular}{|c|c|c|c|}
\hline Parameter & Description & Nominal values & Units \\
\hline$g$ & $\begin{array}{l}\text { Gravitational } \\
\text { acceleration }\end{array}$ & 9.81 & $\mathrm{~m} / \mathrm{s}^{2}$ \\
\hline$m$ & Mass of ball & 0.07 & $\mathrm{~kg}$ \\
\hline$M$ & Mass of beam & 0.15 & $\mathrm{~kg}$ \\
\hline$L$ & Length of beam & 0.4 & $\mathrm{~m}$ \\
\hline$R_{m}$ & $\begin{array}{l}\text { Resistance of } \\
\text { armature of the motor }\end{array}$ & 9 & $\Omega$ \\
\hline$J_{m}$ & $\begin{array}{l}\text { Moment of inertia of } \\
\text { motor }\end{array}$ & $7.35 \times 10^{-4}$ & $\mathrm{Nm} / \mathrm{rad} / \mathrm{s}^{2}$ \\
\hline$C_{m}$ & $\begin{array}{l}\text { Torque constant of } \\
\text { motor }\end{array}$ & 0.0075 & $\mathrm{Nm} / \mathrm{A}$ \\
\hline$C_{g}$ & Gear ratio & 4.28 & - \\
\hline$d$ & $\begin{array}{l}\text { Radius of arm } \\
\text { connected to } \\
\text { servomotor }\end{array}$ & 0.04 & $\mathrm{~m}$ \\
\hline$J_{1}$ & $\begin{array}{l}\text { Moment of inertia of } \\
\text { beam }\end{array}$ & 0.001 & $\mathrm{~kg} \mathrm{~m}^{2}$ \\
\hline$C_{b}$ & $\begin{array}{l}\text { Back emf constant } \\
\text { value }\end{array}$ & 0.5625 & $\mathrm{~V} / \mathrm{rad} / \mathrm{s}$ \\
\hline
\end{tabular}

Now, writing this in the controllable canonical form (phase variable form), one may have

$$
\begin{gathered}
\dot{\xi}_{1}=\xi_{2} \\
\dot{\xi}_{2}=\xi_{3} \\
\vdots \\
\dot{\xi}_{4}=\varphi(\widehat{\xi})+\gamma(\widehat{\xi}) \tau+\gamma(\widehat{\xi}) \Delta G_{m}(\widehat{\xi}, \widehat{\tau}, t),
\end{gathered}
$$

where $y^{(i-1)}=\xi_{i}$,

$$
\begin{aligned}
& \varphi(\widehat{\xi})=\frac{1}{C_{4}\left(m x_{1}^{2}+C_{1}\right)}\left[\left(2 m x_{1} x_{2}+C_{2}\right) x_{4} \cos x_{3}\right. \\
& \quad+\left(m g x_{1}+\frac{L}{2} M g\right) \cos ^{2} x_{3} \\
& \left.\quad+x_{4}^{2}\left(m x_{1}^{2}+C_{1}\right) \cos x_{3}\right]
\end{aligned}
$$

$\gamma(\widehat{\xi}) \tau=-\tau \cos x_{3}$, and $\gamma(\widehat{\xi}) \Delta G_{m}(\widehat{\xi}, \widehat{\tau}, t)$ represents the model uncertainties. Here we discuss ISMC on ball and beam system with fixed step tracking as well as variable step tracking. The integral manifold is defined as follows:

$$
\sigma=c_{1} \xi_{1}+c_{2} \xi_{2}+c_{3} \xi_{3}+\xi_{4}+z
$$

The expression of the overall controller which becomes $\dot{\sigma}$ will be as follows:

$$
\begin{aligned}
\tau_{1}= & -k_{1} \xi_{1}-k_{2} \xi_{2}-k_{3} \xi_{3}-k_{4} \xi_{4} \\
& +\frac{1}{\gamma(\widehat{\xi})}\left(-\varphi(\widehat{\xi})-(\gamma(\widehat{\xi})-1) \tau_{0}-K \operatorname{sign} \sigma\right), \\
\dot{\sigma}= & c_{1} \dot{\xi}_{1}+c_{2} \dot{\xi}_{2}+c_{3} \dot{\xi}_{3}+f_{s}+h_{s} \tau_{0}+h_{s} \tau_{1}+\dot{z} \\
\dot{z}= & -c_{1} x_{2}+\frac{c_{2} g}{C_{4}} \sin x_{3}+\frac{c_{3} g}{C_{4}} x_{4} \cos x_{3}-\gamma(\widehat{\xi}) \tau_{0} \\
& -\varphi(\widehat{\xi}) .
\end{aligned}
$$

As the authors are performing the reference tracking here, therefore, the integral manifold and the controller will appear as follows:

$$
\sigma=c_{1}\left(\xi_{1}-r_{d}\right)+c_{2} \xi_{2}+c_{3} \xi_{3}+\xi_{4}+z
$$

$\tau_{1}$

$$
\begin{aligned}
= & -k_{1}\left(\xi_{1}-r_{d}\right)-k_{2} \xi_{2}-k_{3} \xi_{3}-k_{4} \xi_{4} \\
& +\frac{1}{\gamma(\widehat{\xi})}\left(-\varphi(\widehat{\xi}, \tau)-(\gamma(\widehat{\xi})-1) \tau_{0}-K \operatorname{sign}(\sigma)\right),
\end{aligned}
$$

where $r_{d}$ is the desired reference with $\dot{r_{d}}, \ddot{r_{d}}, \ddot{r_{d}}$ being bounded.

4.3. Simulation Results. The simulation study of the system is carried out by considering the reference tracking of a square wave signal and sinusoidal wave signal. In the subsequent paragraph their respective results will be demonstrated in detail.

In case the efforts are directed to track a fixed square wave signal in the presence of disturbances, the initial conditions of the system were set to $x_{1}(0)=0.4, x_{2}(0)=x_{3}(0)=x_{4}(0)=0$. Furthermore, the square wave was defined in the simulation code as follows:

$$
r_{d}(t)= \begin{cases}20 \mathrm{~cm} & 0 \leq t \leq 19 \\ 14 \mathrm{~cm} & 20 \leq t \leq 39 \\ 20 \mathrm{~cm} & 40 \leq t \leq 60 .\end{cases}
$$

The gains of the proposed controller presented from (39) to (41) are chosen according to Table 2.

The output tracking performance of the proposed control input, when a square wave is used as desired reference output, is shown in Figure 2. It can be clearly examined that the performance is very appealing in this case. The corresponding sliding manifold profile is displayed in Figure 3 which clearly indicates that the sliding mode is established from the very beginning of the processes which in turn results in enhanced robustness. The controlled input signal's profile is depicted in Figure 4 with its zoomed profile as shown in Figure 5. It is obvious from both the figures that the control input derives the system with suppressed chattering phenomenon which is 
TABLE 2: Parametric values used in the square wave tracking.

\begin{tabular}{lcccccccc}
\hline Constants & $C_{1}$ & $C_{2}$ & $C_{3}$ & $K_{1}$ & $K_{2}$ & $K_{3}$ & $K_{4}$ & $K$ \\
\hline Values & 1.2 & 1.2 & 0.11 & 402.98 & 250.18 & 60 & 4.1 & 5 \\
\hline
\end{tabular}

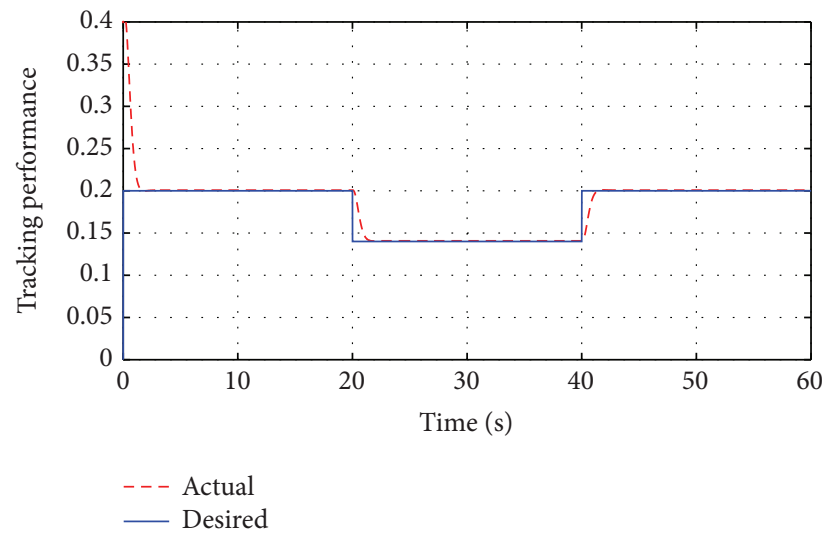

FIGURE 2: Output tracking performance when a square wave is used as reference/desired output.

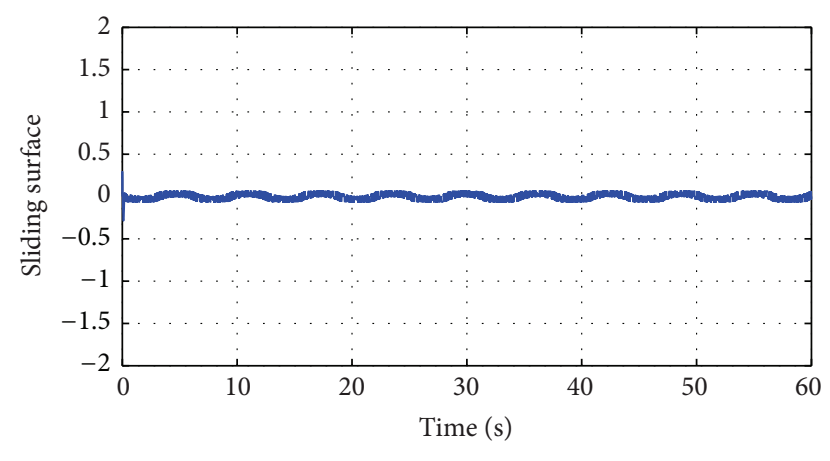

— Sliding surface

FIGURE 3: Sliding manifold convergence profile in case of square wave tracking.

tolerable for the system actuators health. Now, from this case study, it is concluded that integral sliding mode approach is an interesting candidate for this class.

In this case study, once again, efforts are focused on the tracking of a sinusoidal signal, which is defined as $r_{d}(t)=$ $\sin (t)$, in the presence of disturbances. Like the previous case study, the initial conditions of the system were set to $x_{1}(0)=$ $0.4, x_{2}(0)=x_{3}(0)=x_{4}(0)=0$. In addition, the gains of the proposed controller presented in (50) are chosen according to Table 3 .

The output tracking performance of the proposed control input, when a sinusoidal signal is considered as desired reference output, is shown in Figure 6. It can be clearly seen that the performance is excellent in this scenario. The corresponding sliding manifold profile is displayed in Figure 7 which confirms the establishment of sliding modes from the starting instant and, consequently, enhancement of robustness. The controlled input signal's profile is depicted in Figure 8. It is obvious from the figure that the control input
TABLE 3: Parametric values used in the sinusoid wave tracking.

\begin{tabular}{lcccccccc}
\hline Constants & $C_{1}$ & $C_{2}$ & $C_{3}$ & $K_{1}$ & $K_{2}$ & $K_{3}$ & $K_{4}$ & $K$ \\
\hline Values & 1.2 & 1.2 & 0.11 & 402.98 & 250.18 & 230 & 4.9 & 5 \\
\hline
\end{tabular}

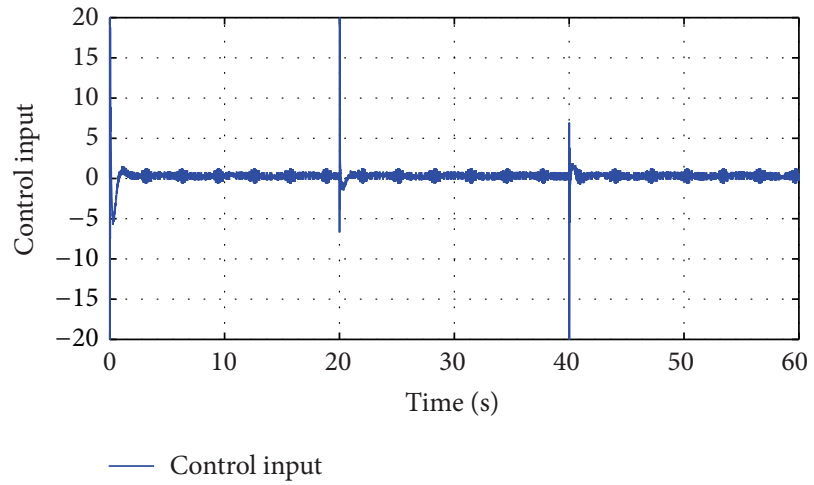

FIGURE 4: Control input in square wave reference tracking.

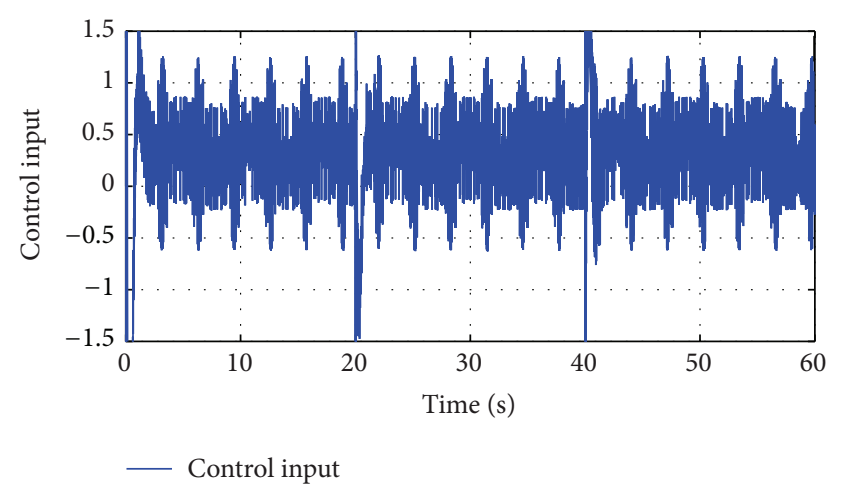

FIGURE 5: Zoom profile of the control input depicted in Figure 4.

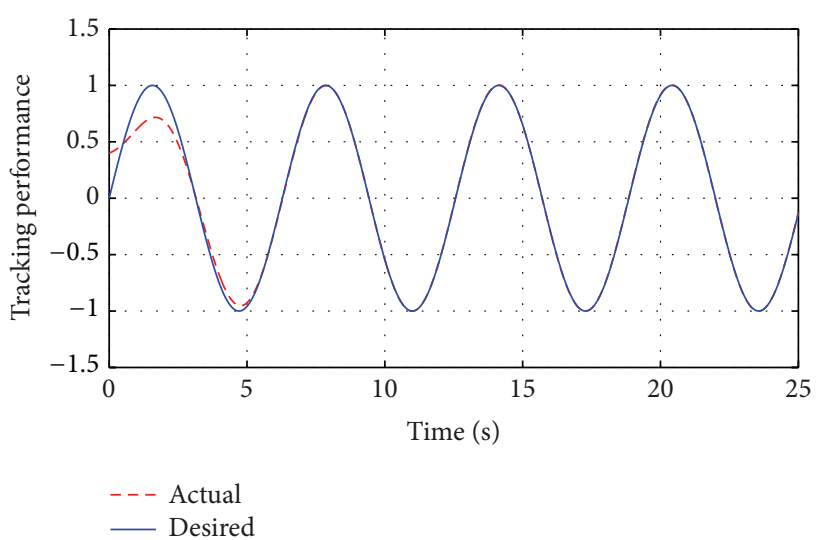

FIGURE 6: Output tracking performance when a sinusoidal wave is used as reference/desired output.

evolves with suppressed chattering phenomenon which, once again, makes this design strategy a good candidate for the class of these underactuated systems.

4.4. Implementation Results. The control technique proposed in this paper is implemented on the actual apparatus using 


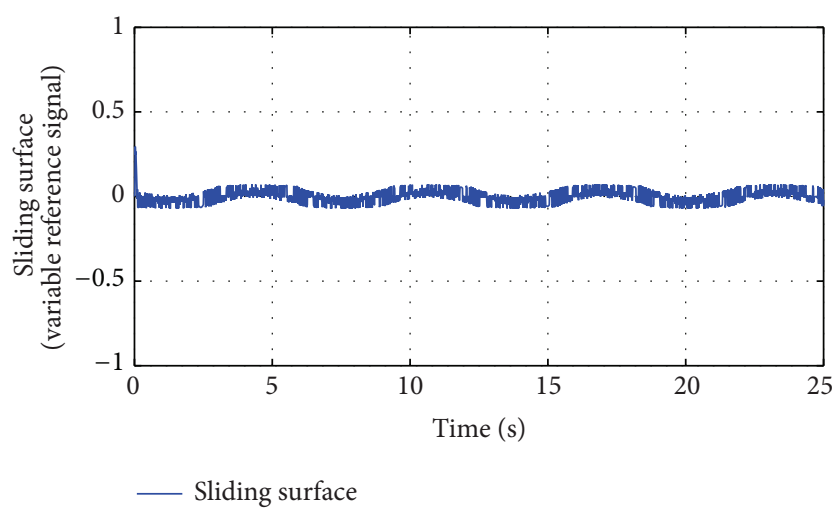

FIGURE 7: Sliding manifold convergence profile in case of sinusoidal wave tracking.

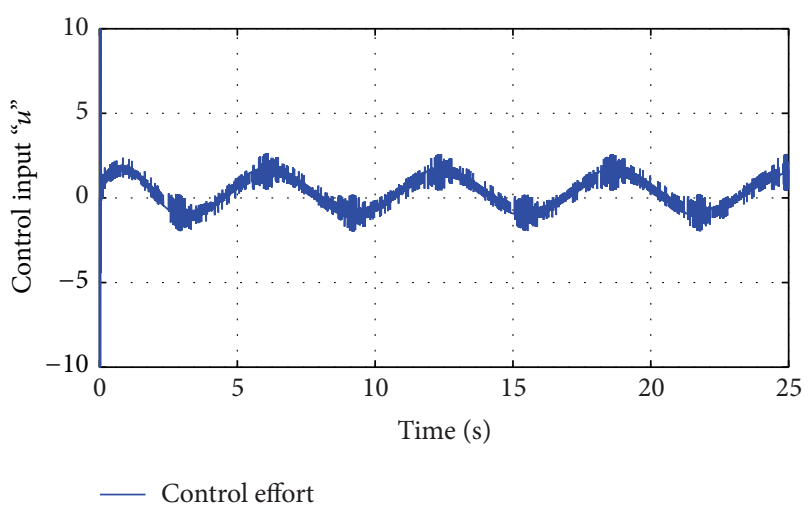

FIgURE 8: Control input in sinusoidal wave reference tracking.

the MATLAB environment. The detailed discussions are presented below.

4.4.1. Experimental Setup Description. The experiment setup is equipped by GoogolTech GBB1004 with an electronic control box. The beam length is $40 \mathrm{~cm}$ along with mass of ball, that is, $28 \mathrm{~g}$, and an intelligent IPM100 servo driver which is used for moving the ball on the beam. The experimental setup is shown in Figure 9.

The input given to apparatus is the voltage $v_{\text {in }}(t)$ and the output is the position of the motor $\theta(t)$, which, in other words, is an input for the positioning of the ball on the beam. This apparatus uses potentiometer mounted within a slot inside the beam to sense the position of the ball on the beam. The measured position along the beam is fed to the $\mathrm{A} / \mathrm{D}$ converter of IPM100 motion drive.

The power module used in GoogolTech requires $220 \mathrm{~V}$ and $10 \mathrm{~A}$ input. Note that the control accuracy of this manufactured apparatus lies within the range of $\pm 1 \mathrm{~mm}$. The typical parameters values are listed in Table 1. The environment used here includes Windows XP as an operating system and MATLAB 7.12/Simulink 7.7. Furthermore, the sampling time used in forthcoming practical results was $2 \mathrm{~ms}$. In the experimental processes, the proposed controllers need velocity measurements which are, in general, not available. One may

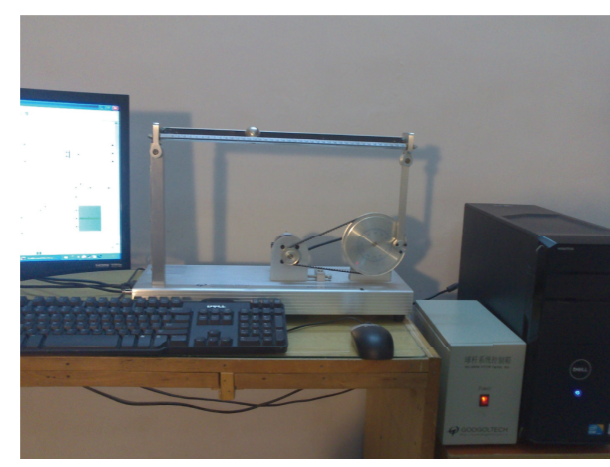

FIGURE 9: Experimental setup of the ball and beam equipped via GoogolTech GBB1004.

use different kind of velocity observers/differentiator for the velocity estimation [16]. In order to make the implementation easy and simple, a derivative block of the Simulink environment is used to provide the corresponding velocities measurements. Now, we are ready to discuss the results of the system.

In this experiment, the initial conditions were set to $x_{1}(0)=0.28, x_{2}(0)=x_{3}(0)=x_{4}(0)=0$. The reference signal which is needed to be tracked is being defined in (51). In Figures 10 and 11, the tracking performance is shown. The results reveal that the actual signal $x_{1}(t)$ is pretty close to the desired signal $r_{d}(t)$ with a steady state error which is approximately $\pm 0.001 \mathrm{~m}$. The existence of this error is because of the apparatus.

The observations of these tracking results make it clear that the practically implemented results have very close resemblance with the simulation result presented in Figure 2. The error convergence depends on the initial conditions of the ball on the beam. If the ball is placed very close to the desired reference value then it will take little time to reach the desired position. On the other hand, the convergence to the desired position will take considerable time if the initial condition is chosen far away from the desired values. This phenomenon of convergence is according to the equipment design and structure.

The sliding manifold convergence and the control input are shown in Figures 12 and 13, respectively. The control input and the sliding manifolds show some deviations in the first second. This deviation occurs because the ball on the beam, being placed anywhere on the beam, is first moved to one side of the beam and then ball moved to the desired position. The zoomed profile of the control input, being displayed in Figure 14, shows high frequency vibration (chattering) of magnitude \pm 0.07 . This makes the proposed control design algorithm an appealing candidate for this class of nonlinear systems. The gains of the controller being used in this experiment are displayed in Table 4.

\section{Conclusion}

The control of underactuated systems, because of their less number of actuators than the degree of freedom, is an 
TABLE 4: Parametric values used in implementation.

\begin{tabular}{lcccccccc}
\hline Constants & $C_{1}$ & $C_{2}$ & $C_{3}$ & $K_{1}$ & $K_{2}$ & $K_{3}$ & $K_{4}$ & $K$ \\
\hline Values & 8 & 5 & 1 & 3 & 15 & 3 & 1 & 4
\end{tabular}

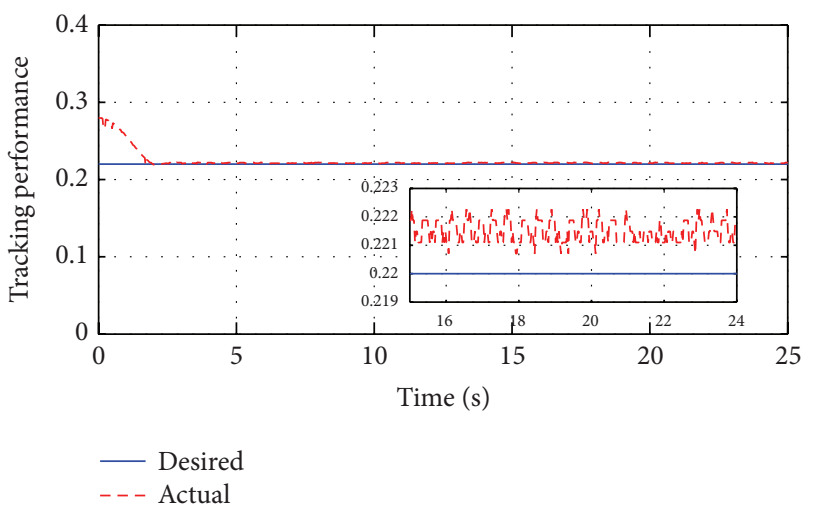

FIGURE 10: Output tracking performance when $r_{d}=22 \mathrm{~cm}$ is set as reference/desired output.

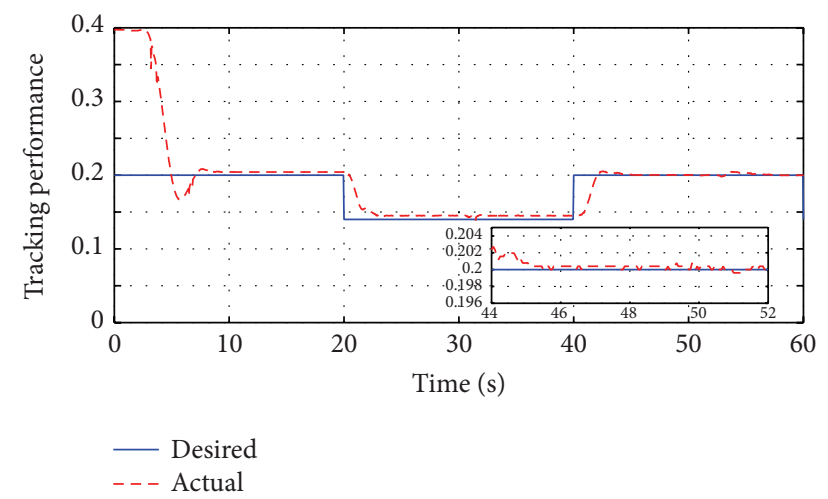

FIGURE 11: Output tracking performance when a square wave is used as reference/desired output.

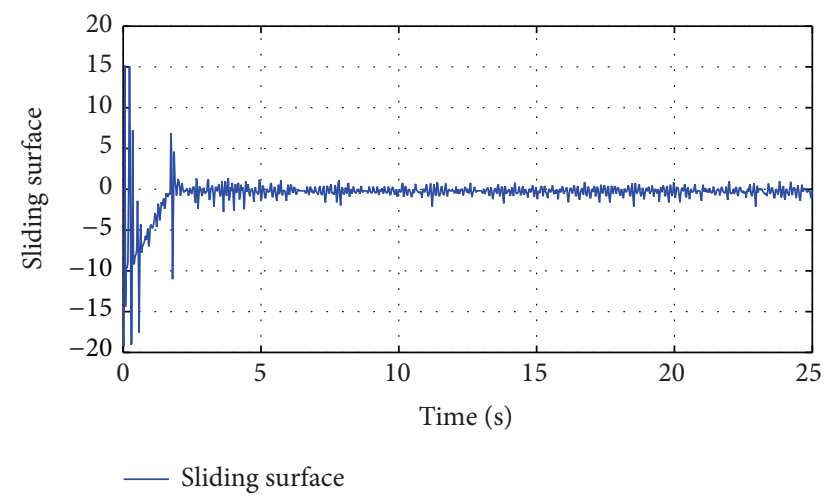

FIGURE 12: Sliding surface of practical system.

interesting objective among the researchers. In this work, an integral sliding mode control approach, due to its robustness from the very beginning of the process, is employed for the control design of this class. The design of the integral manifold relied upon a transformed form. The benefit of the transformed form is that it makes the design strategy easy and

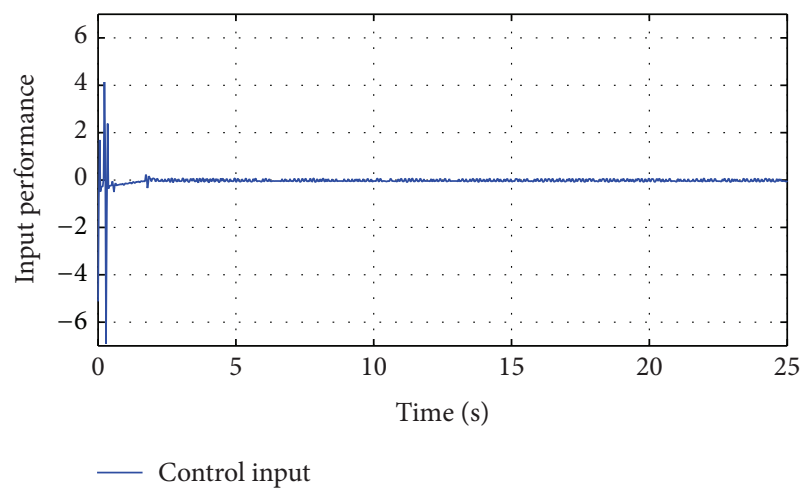

FIGURE 13: Control input for reference tracking.

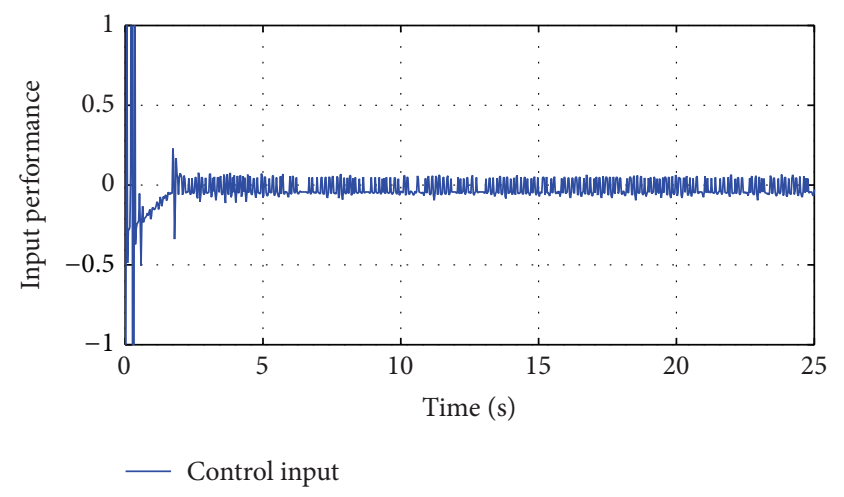

FIGURE 14: Zoom profile of the control input depicted in Figure 13.

simple. The stability analysis and experimental results of the proposed control laws are presented, which convey the good features and demand the proposed approach when the system operates under uncertainties.

\section{Conflict of Interests}

The authors declare that there is no conflict of interests regarding the publication of this paper.

\section{References}

[1] S. Mahjoub, F. Mnif, and N. Derbel, "Set point stabilization of a 2DOF underactuated manipulator," Journal of Computers, vol. 6, no. 2, pp. 368-376, 2011.

[2] F. Mnif, "VSS control for a class of underactuated Mechanical systems," International Journal of Computational Cognition, vol. 3, no. 2, pp. 14-18, 2005.

[3] J. Hauser, S. Sastry, and P. Kokotovic, "Nonlinear control via approximate input-output linearization: the ball and beam example," IEEE Transactions on Automatic Control, vol. 37, no. 3, pp. 392-398, 1992.

[4] M. Jankovic, D. Fontanine, and P. V. Kokotovic, "TORA example: cascade and passitivity-based control designs," IEEE Transactions on Control Systems Technology, vol. 6, pp. 43474351, 1996. 
[5] T. Sugie and K. Fujimoto, "Control of inverted pendulum systems based on approximate linearization: design and experiment," in Proceedings of the 33rd IEEE Conference on Decision and Control, vol. 2, pp. 1647-1648, December 1994.

[6] R. Xu and Ü. Özgüner, "Sliding mode control of a class of underactuated systems," Automatica, vol. 44, no. 1, pp. 233-241, 2008.

[7] M. W. Spong, "The swing-up control problem for the acrobat," IEEE Transactions on Systems Technology, vol. 15, no. 1, pp. 4955, 1995.

[8] R. W. Brockett, "Asymptotic stability feedback and stabilization," in Differential Geometric Control Theory, pp. 181-191, Birkhäuser, Boston, Mass, USA, 1983.

[9] C. J. Tomlin and S. S. Sastry, "Switching through singularities," Systems and Control Letters, vol. 35, no. 3, pp. 145-154, 1998.

[10] W.-H. Chen and D. J. Ballance, "On a switching control scheme for nonlinear systems with ill-defined relative degree," Systems \& Control Letters, vol. 47, no. 2, pp. 159-166, 2002.

[11] F. Zhang and B. Fernndez-Rodriguez, "Feedback linearization control of systems with singularities," in Proceedings of the 6th International Conference on Complex Systems (ICCS '06), Boston, Mass, USA, June 2006.

[12] D. Seto and J. Baillieul, "Control problems in super-articulated mechanical systems," IEEE Transactions on Automatic Control, vol. 39, no. 12, pp. 2442-2453, 1994.

[13] M. W. Spong, Energy Based Control of a Class of Underactuated Mechanical Systems, IFAC World Congress, 1996.

[14] V. I. Utkin, Sliding Mode Control in Electromechanical Systems, Taylor \& Francis, 1999.

[15] M. Rubagotti, A. Estrada, F. Castanos, A. Ferrara, and L. Fridman, "Integral sliding mode control for nonlinear systems with matched and unmatched perturbations," IEEE Transaction on Automatic Control, vol. 56, no. 11, pp. 2699-2704, 2011.

[16] Q. Khan, A. I. Bhatti, S. Iqbal, and M. Iqbal, "Dynamic integral sliding mode for MIMO uncertain nonlinear systems," International Journal of Control, Automation and Systems, vol. 9, no. 1, pp. 151-160, 2011.

[17] R. Olfati-Saber, "Normal forms for underactuated mechanical systems with symmetry," IEEE Transactions on Automatic Control, vol. 47, no. 2, pp. 305-308, 2002.

[18] R. Lozano, I. Fantoni, and D. J. Block, "Stabilization of the inverted pendulum around its homoclinic orbit," Systems and Control Letters, vol. 40, no. 3, pp. 197-204, 2000.

[19] E. Altug, J. P. Ostrowski, and R. Mahony, "Control of a quadrotor helicopter using visual feedback," in Proceedings of the IEEE International Conference on Robotics and Automation (ICRA '02), vol. 1, pp. 72-77, Washington, DC, USA, 2002.

[20] A. Isidori, Nonlinear Control Systems, Communications and Control Engineering Series, Springer, Berlin, Germany, 3rd edition, 1995.

[21] H. Sira-Ramirez, "On the dynamical sliding mode control of nonlinear systems," International Journal of Control, vol. 57, no. 5, pp. 1039-1061, 1993.

[22] Q. Khan, A. I. Bhatti, and A. Ferrara, "Dynamic sliding mode control design based on an integral manifold for nonlinear uncertain systems," Journal of Nonlinear Dynamics, vol. 2014, Article ID 489364, 10 pages, 2014.

[23] C. Edwards and S. K. Spurgeon, Sliding Modes Control: Theory and Applications, Taylor \& Francis, London, UK, 1998.

[24] A. M. Khan, A. I. Bhatti, S. U. Din, and Q. Khan, "Static \& dynamic sliding mode control of ball and beam system," in Proceedings of the 9th International Bhurban Conference on Applied Sciences and Technology (IBCAST '12), pp. 32-36, Islamabad, Pakistan, January 2012.

[25] N. B. Almutairi and M. Zribi, "On the sliding mode control of a Ball on a Beam system," Nonlinear Dynamics, vol. 59, no. 1-2, pp. 222-239, 2010. 


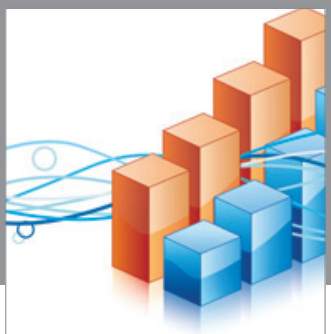

Advances in

Operations Research

vatem alat4

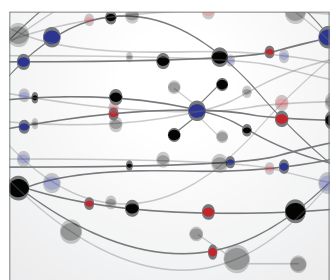

\section{The Scientific} World Journal
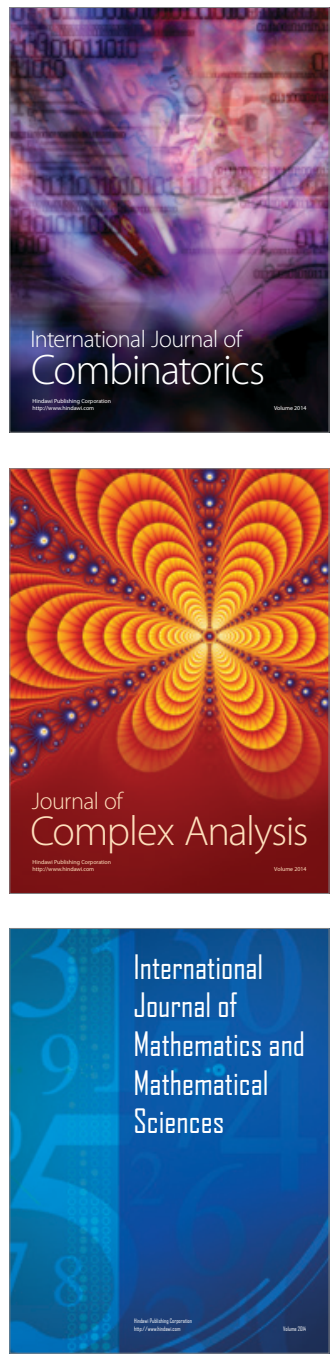
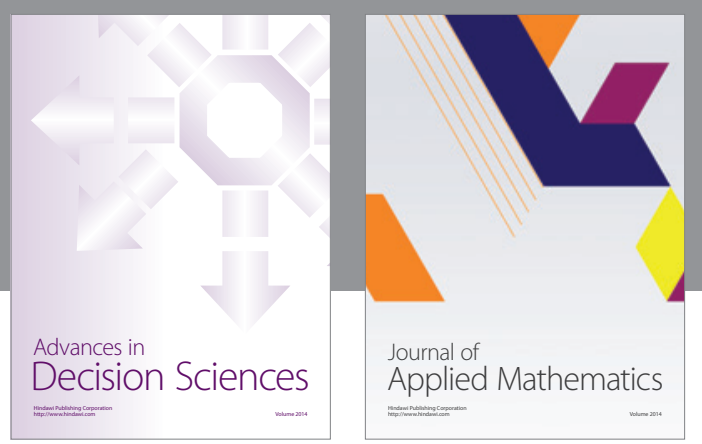

Algebra

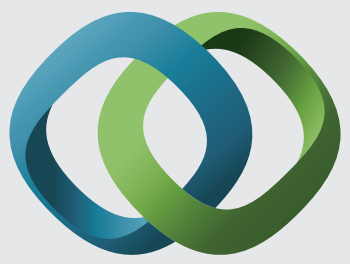

\section{Hindawi}

Submit your manuscripts at

http://www.hindawi.com
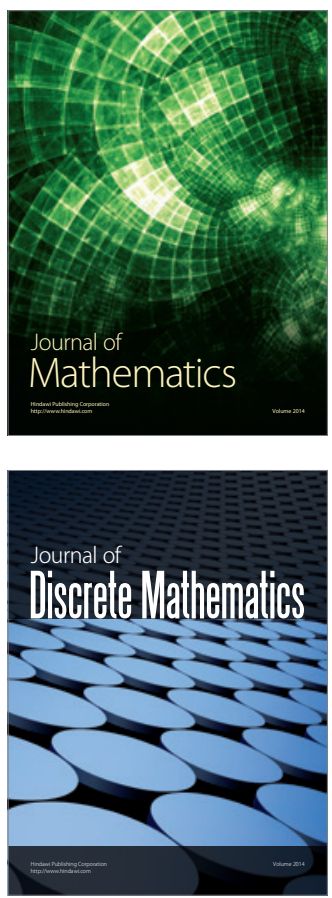

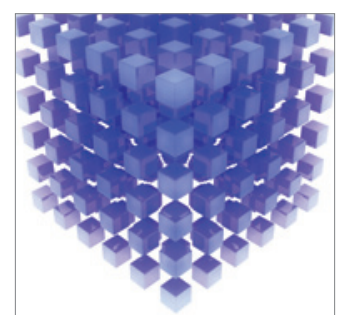

Mathematical Problems in Engineering
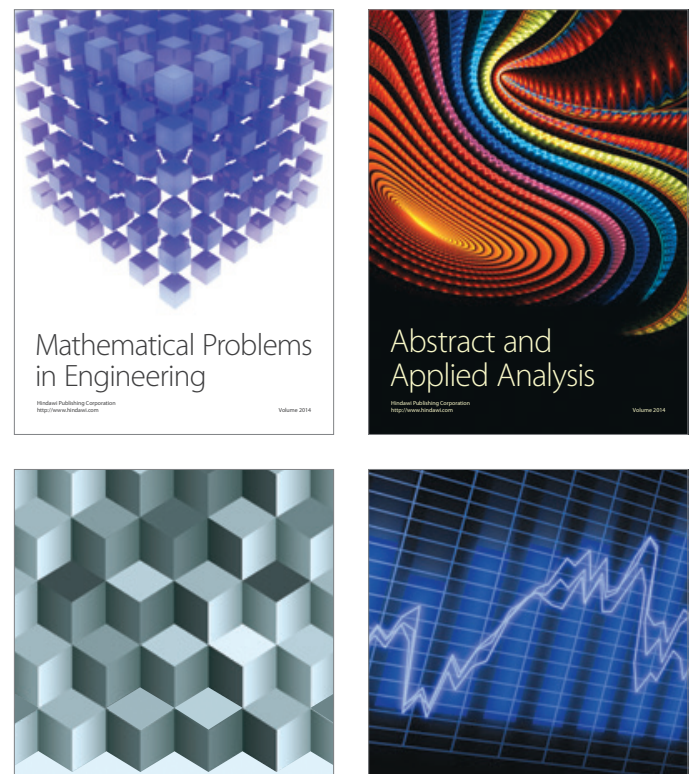

Journal of

Function Spaces

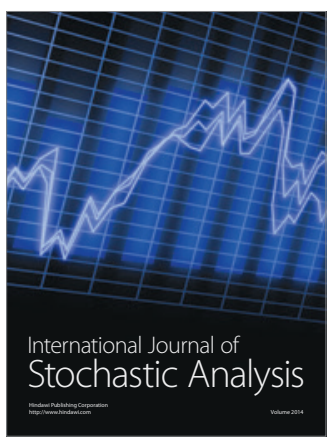

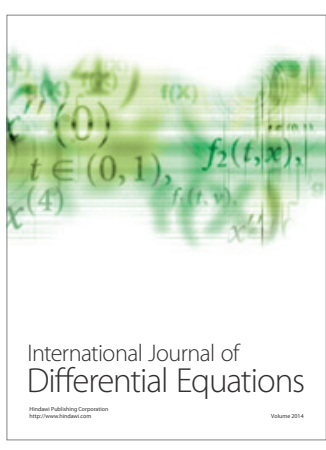
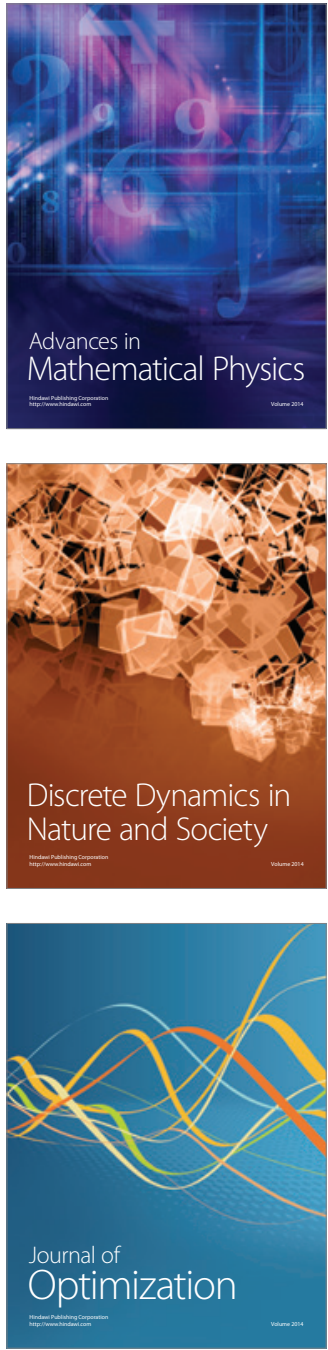